\title{
Imaging the multiple facets of immuno-oncology
}

\author{
Chaitanya Divgi ${ }^{1}$
}

Published online: 8 November 2017

(C) Springer-Verlag GmbH Germany 2017

The emerging field of "immuno-oncology" has revolutionized cancer therapy, with several antibodies demonstrating survival improvements in a variety of cancers [1-3]. The most studied antibodies $[1,2]$ target PD-1, a receptor found on immune cells that bind to a tumor-associated antigen, PD-L1. Antibodies that target PD-L1 have also been successfully employed [3]. Central to the efficacy of agents that target various immune checkpoints has been abrogation of immune cell populations that collectively prevent tumor regression and/or containment [4]. This protection is putatively abrogated by the antibody, enabling cytotoxic immune cells to attack the tumor. These therapies are expensive, can cause significant morbidity, and are not always effective [1-4]. There thus remains a need, largely unmet, to better identify patients who are likely to respond to such therapy.

Checkpoint blockade inhibition therapy is currently undertaken in patients whose tumors exhibit evidence of the putative target; for instance, patients whose tumor cells express PD-L1 (the ligand that is targeted by PD-1) are more likely to respond to anti-PD-1 therapy. Tumor expression of these targets is usually assessed by immunohistochemistry of (frequently historical) samples. It has been demonstrated that there is a relationship between target expression and therapeutic efficacy, as may be expected [5].

Another interesting approach is comprehensively examined in this issue of the journal [6]. Rather than assess the extent of tumor cells bearing the PD-L1 receptor, England et al. quantitatively imaged, in an adequate murine model, the presence and

This Editorial Commentary refers to the article http://doi.org/10.1007/ s00259-017-3803-4

Chaitanya Divgi

crdivgi@gmail.com

1 Divgi Consulting LLC, Meadowbrook, PA 19046, USA extent of the aberrant immune cells that express PD-1. Demonstrating tumor targeting of the therapeutic in this manner would not only have promise as a predictive biomarker for efficacy of anti-PD-1 therapy, it would also serve, potentially, as a pharmacodynamic biomarker for response, perhaps by demonstrating a reduction in PD-1-positive cells in a tumor upon institution of therapy. That would in turn help better select patients for therapy, and also help determine when to discontinue an expensive and not entirely innocuous therapeutic modality.

This report provides a strong underpinning to the clinical trials that currently evaluate PET/CT using (similar) macromolecules that target PD-1. Central to their success is, of course, the assumption that PD-1 is expressed only in the culprit peri-tumoral immune cells, a finding borne out by several investigators [7]. Most importantly, it is intuitive that targeting of a therapeutic to its target would be an important predictor of its efficacy.

The current standard remains assessment of PD-L1 expression in tumor cells in representative tumor tissue [1,2]. There, nonetheless, remains an unmet need for better prediction of response to checkpoint blockade immunotherapy $[8,9]$. Other groups have accordingly focused on other aspects of the immuno-oncologic spectrum. Antibodies as well as small molecules that target PD-L1 have been developed and are being studied in animal models as well as in the clinic [10, 11]. Clinical trials are also currently on-going with a radiolabeled minibody that targets CD8-positive cells [12], whose recruitment to the tumor is believed critical to successful therapy. Other putative cytokines expressed by leukocyte cell populations that are recruited to the tumor site (upon successful blockade of the checkpoint), including particularly granzyme B [13], expressed by activated macrophages, are also being evaluated in preparation for clinical study.

All these efforts are directed toward utilizing molecular imaging as a biomarker, in this instance, whole-body imaging. 
We may safely assume that in a patient with metastatic disease, there will be varying expression of the putative biomarker among the metastases (and perhaps within a metastasis). Thus, determination of what constitutes a positive result will be critical.

Immunohistochemistry, used as a predictive biomarker, utilizes a number of continuous output variables, including number of stained cells as well as intensity of staining, in what is generally described as an "average high-power field", toward a dichotomous positive or negative output. Imaging would be analogous but applied across multiple lesions throughout the body, and thus with far more continuous variables. One will need to decide whether and how to grade the positivity (by SUV? as a ratio to a normal organ?) within a lesion; one will further need to decide whether all measurable/evaluable lesions should be included in the analysis, or whether, analogous to RECIST [14], a critical number of lesions in defined sites would be chosen for assessment. Accordingly, one would embark on the development of the imaging biomarker with all these parameters being addressed from the very first "carefully controlled clinical trial". That would further mean the incorporation of imaging biomarker studies early in drug development, where they may be verified as exploratory end-points before being validated as predictive biomarkers in later phase trials.

The ability to image the entire body increases our ability to understand disseminated disease processes such as cancer in a very comprehensive manner. Attendant to that is a seeming excess of information. This information abundance is to be harnessed not feared. Where knowledge is concerned, there can never be too much of a good thing. The path being cleared by England et al., and others in the pursuit of a useful imaging biomarker for checkpoint-directed immunotherapy, will at the very least provide meaningful pathophysiologic information, and hopefully provide imaging tools that better guide an expensive and not innocuous therapy.

\section{References}

1. Larkin J, Minor D, D'Angelo S, et al. Overall survival in patients with advanced melanoma who received nivolumab versus investigator's choice chemotherapy in CheckMate 037: a randomized, controlled, open-label phase III trial. J Clin Oncol. 2017; https:// doi.org/10.1200/JCO.2016.71.8023.

2. Reck M, Rodríguez-Abreu D, Robinson AG, et al. Pembrolizumab versus chemotherapy for PD-L1-positive non-small-cell lung cancer. N Engl J Med. 2016;375(19):1823-33.

3. Balar AV, Galsky MD, Rosenberg JE. Et al; IMvigor210 study group. Atezolizumab as first-line treatment in cisplatin-ineligible patients with locally advanced and metastatic urothelial carcinoma: a single-arm, multicentre, phase 2 trial. Lancet. 2017;389(10064): 67-76. https://doi.org/10.1016/S0140-6736(16)32455-2.

4. Sharma P, Allison JP. The future of immune checkpoint therapy. Science. 2015;348(6230):56-61. https://doi.org/10.1126/science. aaa8172.

5. Chen PL, Roh W, Reuben A, et al. Analysis of immune signatures in longitudinal tumor samples yields insight into biomarkers of response and mechanisms of resistance to immune checkpoint blockade. Cancer Discov. 2016;6(8):827-37. https://doi.org/10. 1158/2159-8290.CD-15-1545.

6. England CG, Jiang D, Ehlerding EB, Rekoske BT, Ellison PA, Hernandez R, et al. ${ }^{89} \mathrm{Zr}$-labeled nivolumab for imaging of T-cell infiltration in a humanized murine model of lung cancer. Eur J Nucl Med Mol Imaging. 2017. https://doi.org/10.1007/s00259-0173803-4.

7. Chen DS, Mellman I. Elements of cancer immunity and the cancerimmune set point. Nature. 2017;541(7637):321-30. https://doi.org/ 10.1038/nature21349. Review

8. Gibney GT, Weiner LM, Atkins MB. Predictive biomarkers for checkpoint inhibitor-based immunotherapy. Lancet Oncol. 2016;17(12):e542-51. https://doi.org/10.1016/S1470-2045(16) 30406-5. Review

9. Meng X, Huang Z, Teng F, Xing L, Yu J. Predictive biomarkers in PD-1/PD-L1 checkpoint blockade immunotherapy. Cancer Treat Rev. 2015;41(10):868-76. https://doi.org/10.1016/j.ctrv.2015.11. 001.

10. Heskamp S, Hobo W, Molkenboer-Kuenen JD, et al. Noninvasive imaging of tumor PD-L1 expression using Radiolabeled anti-PDL1 antibodies. Cancer Res. 2015;75(14):2928-36. https://doi.org/ 10.1158/0008-5472. CAN-14-3477.

11. Donnelly DJ, Smith RA, Morin P, et al. Synthesis and biological evaluation of a novel (18)F-labeled Adnectin as a PET Radioligand for imaging PD-L1 expression. J Nucl Med. 2017; https://doi.org/ 10.2967/jnumed.117.199596.

12. Tavaré R, McCracken MN, Zettlitz KA, et al. Engineered antibody fragments for immuno-PET imaging of endogenous CD8+ T cells in vivo. Proc Natl Acad Sci U S A. 2014;111(3):1108-13. https:// doi.org/10.1073/pnas.1316922111.

13. Larimer BM, Wehrenberg-Klee E, Dubois F, et al. Granzyme B PET imaging as a predictive biomarker of immunotherapy response. Cancer Res. 2017;77(9):2318-27. https://doi.org/10.1158/ 0008-5472.CAN-16-3346.

14. Eisenhauer EA, Therasse P, Bogaerts J, et al. New response evaluation criteria in solid tumours: revised RECIST guideline (version 1.1). Eur J Cancer. 2009;45(2):228-47. https://doi.org/10.1016/j. ejca.2008.10.026. 